Artículo Original

\title{
Hallazgos de videofluoroscopía en niños de 3 a 6 años con parálisis cerebral
}

\author{
JOSÉ LUIS BACCO R ${ }^{1}$, FANNY ARAYA C ${ }^{2}$,
}

NATALIA PEÑA $\mathrm{J}^{3}$, JENNIFFER GARCÍA $\mathrm{R}^{3}$.

\footnotetext{
'Unidad Médica, Instituto Teletón Valparaíso, Chile. ¿Unidad de Fonoaudiología, Instituto Teletón Valparaíso,

3Unidad de Terapia

Ocupacional, Instituto Teletón Valparaíso, Chile.

Correspondencia a: José Luis Bacco R.

e-mail: jbacco@teleton.cl

Recibido: 29-01-2019 Aceptado: 26-04-2019
}

\section{ABSTRACT \\ Videofluoroscopic findings in children from 3 to 6 years old with cerebral palsy}

Introduction: Cerebral palsy $(\mathrm{CP})$ is frequently accompanied by feeding and swallowing disorders (FSD). An early diagnosis of FSD prevents numerous complications. Clinical assessment stands as the first approach to FSD, although it is insufficient to identify risk factors (laryngeal penetration and aspiration). For this purpose, a videofluoroscopic (VFC) evaluation is required. Objective: To describe and quantify VFC findings in a group of children with $\mathrm{CP}$, aged 3 to 6 years, GMFCS I to V. Patients and Methods: 50 patients underwent a VFC after their Gross Motor Functional Classification System (GMFCS) level was determined and no contraindications for the exam were identified. In addition, the researchers conducted an assessment to establish the presence of signs of unsafe and incompetent swallowing, food consistency tolerance, and mealtime positioning requirements, in order to allow a more comfortable VFC procedure. Results: There was male predominance, average age of 5 years, $78 \%$ GMFCS III-V. Up to $94 \%$ had one or more VFC alterations at some point of the exam. $28 \%$ had no chewing ability, being all the cases GMFCS V. Considering the deglutition efficacy, the commonest alteration was residue in the vallecula $(86 \%)$, while the most frequent for swallowing safety was posterior spillage in the oral phase (68\%). $26 \%$ presented silent tracheal aspiration. In the esophageal phase the findings were poor esophageal transit $(30 \%)$ and gastroesophageal reflux (8\%). Conclusions: VFC identified potentially risks in every GMFCS level of children with CP. Some of the VFC alterations are silent, even in mild motor impairment cases.

Key words: Feeding, swallowing, cerebral palsy, videofluoroscopy.

\section{RESUMEN}

Introducción: Los trastornos de la alimentación y deglución (TAD) acompañan frecuentemente a la parálisis cerebral (PC). Su diagnóstico oportuno previene numerosas complicaciones. La evaluación clínica es el primer acercamiento a los TAD, pero resulta insuficiente para identificar situaciones de riesgo (penetración laríngea o aspiración). Para ello, es necesaria una eva- 
luación instrumental como la videofluoroscopía (VFC). Objetivo: Describir y cuantificar los hallazgos de VFC en un grupo de niños con PC, entre 3 años y 6 años 11 meses, GMFCS I-V. Pacientes y Métodos: Se practicó una VFC a 50 pacientes con PC, previa determinación del nivel Gross Motor Functional Classification System (GMFCS) e inexistencia de contraindicaciones para dicho examen. Además, se determinaron signos de incompetencia e inseguridad deglutoria, consistencias toleradas y requerimientos de posicionamiento, para facilitar el procedimiento. Resultados: Población predominantemente masculina, edad promedio de 5 años, $78 \%$ GMFCS III-V. Hasta el 94\% tuvo una o más alteraciones videofluoroscópicas en alguna etapa del examen. El $28 \%$ no presentó capacidad de masticación, siendo todos GMFCS V. En eficacia deglutoria la alteración más frecuente fue el residuo vallecular $(86 \%)$. En seguridad deglutoria fue el derrame posterior en la fase oral (68\%). El $26 \%$ tuvo aspiración traqueal silente. En la fase esofágica, el 30\% presentó tránsito esofágico lento y el $8 \%$ reflujo gastroesofágico. Conclusión: La VFC identificó alteraciones potencialmente riesgosas en todos los niveles GMFCS en niños portadores de PC, algunas de ellas silentes, incluso en casos de compromiso motor leve.

Palabras clave: Alimentación, deglución, parálisis cerebral, videofluoroscopía.

\section{Introducción}

La parálisis cerebral (PC) constituye el cuadro pediátrico discapacitante más frecuente $^{1}$. Definida como un grupo de trastornos de movimiento y postura que limitan la actividad, responde a lesiones en un cerebro inmaduro fetal o infantil ${ }^{2}$. Su prevalencia mundial alcanza 2-3,5 por 1.000 recién nacidos vivos ${ }^{3} y$ se acompaña de numerosas comorbilidades ${ }^{4}$, destacando los trastornos de la alimentación y deglución (TAD) por su alta prevalencia, la que oscila entre $50 \%{ }^{5}$ y $90 \% \%^{6}$. Debido a un TAD, 1 de 15 niños con PC terminará alimentándose por vía no oral en su vida. ${ }^{7}$ Por otra parte, los fenómenos de penetración y aspiración de los TAD contribuyen en forma importante a morbimortalidad respiratoria ${ }^{4-6}$, especialmente complicaciones severas por obstrucción bronquial e infecciones pulmonares recurrentes $^{8,9}$. La neumonía por aspiración es una causa principal de hospitalización en estos pacientes, aunque muchas veces la etiología aspirativa es solamente presunta ${ }^{10}$. En niños con PC, estudios videofluoroscópicos han mostrado una incidencia general de aspiración del $26 \%{ }^{11}$, mientras que la aspiración silente alcanza el $68 \%$ en las formas espásticas severas y alrededor de un tercio de los pacientes ha sufrido una infección pulmonar en los 6 meses previos ${ }^{12}$. En una investigación reciente, se informó que el 34,7\% de un grupo de 49 niños con PC y TAD tenía antecedentes de al menos un cuadro respiratorio aspirativo en los 6 meses anteriores al estudio ${ }^{13}$. La aspiración silente es un problema grave en PC, no sólo causando infecciones respiratorias agudas, sino que también daño inflamatorio pulmonar crónico ${ }^{11}$. Dado que la principal causa de mortalidad en PC es de origen respiratorio ${ }^{14}$, el diagnóstico oportuno de un TAD es imperativo, considerándose como una estrategia que previene y reduce complicaciones en esta condición ${ }^{15}$.

La evaluación de un TAD comienza con un análisis clínico ${ }^{16,17}$, aunque éste no siempre identifica problemas deglutorios. Ello no implica que no existan. Síntomas y signos como tos, atragantamiento, cianosis, voz húmeda y auscultación cervical alterada, entre otros, permiten sospechar afectación de la fase faríngea $^{18,19}$, pero no siempre están presentes. Un reporte reciente indica que la evaluación clínica, si bien muestra alta sensibilidad para detectar 
signos de penetración laríngea y aspiración, tiene una baja especificidad ${ }^{20}$. Sin embargo, complementarla con un estudio instrumental como la videofluoroscopía (VFC) optimiza el diagnóstico de disfagia ${ }^{21}$. La evaluación instrumental de un TAD incluye: la VFC ${ }^{18,22-24}$, el estudio endoscópico clásico o FEES (Fiberoptic Endoscopic Evaluation of Swallowing $)^{22,24-26} \mathrm{y}$ el estudio endoscópico con estimulación para determinar el umbral sensitivo laringofaríngeo o FEES-ST (Fiberoptic Endoscopic Evaluation of Swallowing with Sensory Testing) ${ }^{8}$. La VFC es considerada el gold standard como método de valoración en un $\mathrm{TAD}^{21,22,25}$, siendo el examen instrumental más difundido y de más fácil acceso. Además, entrega información dinámica ordenada secuencialmente por fase deglutoria ${ }^{27}$. A pesar de estos aspectos positivos, la VFC tiene algunas desventajas como ser un test de duración limitada y que no simula una comida en ambiente real, la necesidad de cooperación del paciente y la exposición a la radiación ${ }^{24,28}$. Se ha mencionado también la posibilidad de neumonías por aspiración luego de la VFC, pero un estudio reciente con más de 100 niños derivados a dicho examen no mostró complicaciones infecciosas en ninguno ${ }^{29}$. Algunos autores destacan que la VFC puede resultar un examen particularmente desagradable y traumático en niños ${ }^{30}$, por ello debe realizarse en centros especializados. Aunque la VFC nunca es la evaluación inicial en un $\mathrm{TAD}^{31}$, forma parte importante de las herramientas que permiten objetivar numerosos problemas deglutorios, especialmente si se complementa con la FEES ${ }^{32}$. Las indicaciones de VFC han sido claramente establecidas para su uso racional en $\mathrm{PC}^{31}$, como también los marcadores radiológicos de anormalidad ${ }^{17}$.

Un $35 \%$ de pacientes en los Institutos Teletón tiene $\mathrm{PC} \mathrm{y}$, según un estudio reciente que incluyó a 50 niños con esa condición, hasta un $98 \%$ presenta alguna manifestación clínica de $\mathrm{TAD}^{33}$. Todo lo anterior torna relevante explorar videofluoroscópicamente los casos de TAD y reconocer las alteraciones que arroja. El objetivo del presente estudio es describir y cuantificar los hallazgos de VFC en un grupo de niños con $P C$, entre 3 años y 6 años 11 meses, GMFCS I-V.

\section{Pacientes y Métodos}

\section{Pacientes}

Pacientes con PC, GMFCS I-V, de 3 años a 6 años 11 meses, a abril de 2010, del Instituto Teletón Valparaíso. De un total de 121 niños con esas características, se excluyeron 13 por tener gastrostomía, 7 por comorbilidades descompensadas y 6 por ruralidad. Finalmente, el universo fue de 95 pacientes, del cual se tomó una muestra intencionada de 50 niños $(52,6 \%)$, estratificada por edad.

\section{Metodología}

Los pacientes fueron evaluados individualmente por fisiatra, determinándose el nivel motor grueso según GMFCS (Gross Motor Functional Classification System) ${ }^{2}$, y la factibilidad de indicación de VFC según criterios de la American Speech-LanguageHearing Association (ASHA) ${ }^{34}$ y otras publicaciones recientes ${ }^{17}$. Luego fueron evaluados por fonoaudióloga y terapeuta ocupacional, siguiendo recomendaciones de la $\mathrm{ASHA}^{35}$, y estableciendo presencia de predictores de ineficacia (incoordinación de válvulas deglutorias) e inseguridad (aspiración a vía aérea), tipo de consistencias toleradas y requerimientos de posicionamiento según tono axial. La fonoaudióloga, adicionalmente, determinó quién tenía capacidad masticatoria. Toda esta información se entregaba al operador de la VFC a través de una interconsulta. Todos los pacientes de la muestra fueron elegibles para VFC.

La VFC fue ejecutada en el Hospital Clínico de la Pontificia Universidad Católica de Chile en Santiago por un médico radiólogo, siguiendo protocolos estandarizados ${ }^{36}$. El examen incluyó texturas sólidas, espesas y líquidas. Las sólidas sólo fueron consideradas en pacientes que masticaban. La proyección estándar del examen fue la lateral, agregándose visión frontal en niños con masticación.

Posteriormente, el video del examen y su informe fueron revisados por los investigadores aplicando pautas basadas en diferentes autores ${ }^{17,23,37}$, analizándose presencia general de alteraciones videofluoroscópicas (AV), alteraciones de la masticación de sólidos en quienes tenían dicha función (Cámpora ${ }^{38}$ ) efi- 
cacia deglutoria (adecuado control del bolo y su correcta propulsión) y seguridad deglutoria (ausencia de penetración/aspiración) con la escala de Rosenbek ${ }^{39}$. Se calculó la distribución según GMFCS para todas esas alteraciones $y$, para la eficacia y seguridad deglutorias, se consideró también texturas.

Se elaboró base de datos en planilla Excel, procesándose mediante programa estadístico SPSS, versión 17.0. Se calcularon sólo medidas de posición, dado el carácter cualitativo de las variables.

\section{Aspectos éticos}

Estudio aprobado por la Dirección de Investigación, Docencia y Extensión de Teletón, según la normativa vigente a la fecha de ejecución. Cada participante contó con consentimiento informado.

\section{Resultados}

\section{a) Características epidemiológicas y clínicas}

El promedio etario fue 5 años, con predominio masculino $(56 \%)$ y de casos GMFCS III-V (78\%). El $72 \%$ podía masticar y su VFC incluyó sólidos. El 28\% no podía hacerlo, todos GMFCS V (Tabla 1).

\section{b) Hallazgos en la VFC}

1. Presencia general de AV: Hasta el 94\% presentó una o más anomalías en alguna etapa del examen, viéndose más afectada la eficacia deglutoria.

2. Fase preparatoria oral (Tabla 2): El trastorno más frecuente en el grupo GMFCS I-II fue 'masticación sin sello labial' (36,4\%). También lo fue en el grupo GMFCS III-V $(46,2 \%)$, seguida de 'ausencia de patrón rotatorio' (41\%) y 'alteración de movimientos linguales' $(38,5 \%)$. Los con una o más alteraciones constituyeron el 46,2\% de los casos GMFCS III-V.

3. Eficacia deglutoria (Tabla 3): La alteración específica más frecuente en la fase oral fue 'residuo vallecular', en la categoría 'líquidos y espesos', afectando al 54,5\% del grupo GMFCS I-II y al $84,6 \%$ del

Tabla 1. Características epidemiológicas y clínicas

\begin{tabular}{|lcc|}
\hline Característica & $\mathbf{n}$ & \% \\
Total & 50 & 100 \\
Sexo & & \\
Masculino & 28 & 56 \\
Femenino & 22 & 44 \\
Edad & & \\
3,0-3,9 & 16 & 32 \\
4,0-4,9 & 7 & 24 \\
$5,0-5,9$ & 9 & 28 \\
6,0-6,9 & 18 & 36 \\
Promedio \pm DS (años) & & \\
GMFCS & & \\
I & 3 & 6 \\
II & 3 & 16 \\
III & 8 & 18 \\
IV & 9 & 22 \\
V & 11 & 38 \\
Masticación & 19 & 72 \\
Sí & & 28 \\
No & 36 & \\
\hline
\end{tabular}

Tabla 2. Alteraciones de la fase preparatoria oral (masticación)

\begin{tabular}{|lcccccc|}
\hline Tipo de alteración & \multicolumn{2}{c}{ GMFCS I-II } & \multicolumn{2}{c}{ GMFCS III-V } & \multicolumn{2}{c|}{ Total } \\
& $\mathbf{n = 1 1}$ & $\mathbf{\%}$ & $\mathbf{n = 3 9}$ & $\mathbf{\%}$ & $\mathbf{n = 5 0}$ & $\mathbf{5}$ \\
Masticación no evaluable & - & - & 14 & 35,9 & 14 & 28 \\
Corte inefectivo & 1 & 9,1 & 11 & 28,2 & 12 & 24 \\
\hline Ausencia de patrón rotatorio & 1 & 9,1 & 16 & 41 & 17 & 34 \\
Masticación sin sello labial & 4 & 36,4 & 18 & 46,2 & 22 & 44 \\
\hline Movimientos linguales alterados en masticación & 1 & 9,1 & 15 & 38,5 & 16 & 32 \\
\hline Total con una o más alteraciones & 4 & 36,4 & 18 & 46,2 & 22 & 44 \\
\hline
\end{tabular}


grupo GMFCS III-V. En la fase faríngea, la alteración 'residuos en senos piriformes' se presentó en el 54,5\% del grupo GMFCS I-II y el 76,9\% del grupo GMFCS III-V.

4. Seguridad deglutoria (Tabla 4): La fase oral presentó alta frecuencia de inseguridad por derrame posterior, predominando en niños GMFCS III-V $(64,1 \%)$ para la categoría 'líquidos y espesos'. La fase faríngea fue insegura por fenómenos de penetración, destacando la 'penetración supraglótica con barrido' para la categoría 'espesos' en el grupo GMFCS III-V (17,9\%), y por aspiración, con un 18,2\% de 'aspiración traqueal silente' para líquidos en el grupo GMFCS I-II y un $17,9 \%$ para la categoría 'líquidos y espesos' en el grupo GMFCS III-V.

Tabla 3. Alteraciones de la eficacia deglutoria

\begin{tabular}{|c|c|c|c|c|c|c|c|c|c|c|c|c|c|c|}
\hline \multirow[t]{3}{*}{ Fase } & \multicolumn{4}{|c|}{ Sólo líquidos } & \multicolumn{4}{|c|}{ Sólo espesos } & \multicolumn{4}{|c|}{ Líquidos + espesos } & \multicolumn{2}{|c|}{ Total } \\
\hline & \multicolumn{2}{|c|}{ GMFCS I-II } & \multicolumn{2}{|c|}{ GMFCS III-V } & \multicolumn{2}{|c|}{ GMFCS I-II } & \multicolumn{2}{|c|}{ GMFCS III-V } & \multicolumn{2}{|c|}{ GMFCS I-II } & \multicolumn{2}{|c|}{ GMFCS III-IV } & \multirow[b]{2}{*}{$n=\mathbf{5 0}$} & \multirow[b]{2}{*}{$\%$} \\
\hline & $\mathrm{n}=11$ & $\%$ & $n=39$ & $\%$ & $n=11$ & $\%$ & $n=39$ & $\%$ & $n=11$ & $\%$ & $n=39$ & $\%$ & & \\
\hline \multicolumn{15}{|l|}{ Fase oral } \\
\hline $\begin{array}{l}\text { Sello labial incompleto más } \\
\text { derrame anterior }\end{array}$ & 1 & 9,1 & 1 & 2,6 & - & - & & - & - & - & 16 & 41 & 18 & 36,0 \\
\hline $\begin{array}{l}\text { Alteración del cierre velofaríngeo } \\
\text { con reflujo faringonasal }\end{array}$ & 1 & 9,1 & 4 & 10,3 & - & - & & - & - & - & 5 & 12,8 & 10 & 20,0 \\
\hline $\begin{array}{l}\text { Alteración de sello velolingual } \\
\text { (propulsión múltiple) }\end{array}$ & - & - & 1 & 2,6 & - & - & & - & 1 & 9,1 & 22 & 56,4 & 24 & 48,0 \\
\hline Residuo vallecular & 2 & 18,2 & - & - & - & - & 2 & 5,1 & 6 & 54,5 & 33 & 84,6 & 43 & 86,0 \\
\hline \multicolumn{15}{|l|}{ Fase faríngea } \\
\hline Residuos en senos piriformes & 2 & 18,2 & 3 & 7,7 & - & - & 1 & 2,6 & 6 & 54,5 & 30 & 76,9 & 42 & 84,0 \\
\hline Total con 1 o más alteraciones & 2 & 18,2 & 4 & 10,3 & - & - & 2 & 2,6 & 6 & 54,5 & 33 & 84,6 & 47 & 94,0 \\
\hline
\end{tabular}

Tabla 4. Alteraciones de la seguridad deglutoria

\begin{tabular}{|c|c|c|c|c|c|c|c|c|c|c|c|c|c|c|}
\hline \multirow[t]{3}{*}{ Alteración } & \multicolumn{4}{|c|}{ Líquidos } & \multicolumn{4}{|c|}{ Espesos } & \multicolumn{4}{|c|}{ Líquidos + espesos } & \multirow{3}{*}{$n=50$} & \multirow[b]{3}{*}{$\%$} \\
\hline & \multicolumn{2}{|c|}{ GMFCS I-II } & \multicolumn{2}{|c|}{ GMFCS III-V } & \multicolumn{2}{|c|}{ GMFCS I-II } & \multicolumn{6}{|c|}{ GMFCS III-V GMFCS I-II GMFCS III-IV } & & \\
\hline & $n=11$ & $\%$ & $\mathrm{n}=39$ & $\%$ & $n=11$ & $\%$ & $\mathbf{n}=39$ & $\%$ & $n=11$ & $\%$ & $n=39$ & $\%$ & & \\
\hline \multicolumn{15}{|l|}{ Fase oral } \\
\hline Derrame posterior & 1 & 9,1 & 3 & 7,7 & - & - & 1 & 2,6 & 4 & 36,4 & 25 & 64,1 & 34 & 68,0 \\
\hline \multicolumn{15}{|l|}{ Fase faríngea } \\
\hline $\begin{array}{l}\text { Penetración supraglótica con } \\
\text { barrido }\end{array}$ & - & - & - & - & - & - & 7 & 17,9 & 1 & 9,1 & 5 & 12,8 & 13 & 26,0 \\
\hline $\begin{array}{l}\text { Penetración laríngea sin barrido } \\
\text { funcional }\end{array}$ & - & - & 3 & 7,7 & - & - & - & - & - & - & - & - & 3 & 6,0 \\
\hline $\begin{array}{l}\text { Penetración laríngea hasta } \\
\text { cuerdas vocales con tos }\end{array}$ & 1 & 9,1 & 1 & 2,6 & - & - & - & - & 1 & 9,1 & 2 & 5,1 & 5 & 10,0 \\
\hline $\begin{array}{l}\text { Penetración laríngea hasta } \\
\text { cuerdas vocales sin tos }\end{array}$ & 1 & 9,1 & 3 & 7,7 & - & - & - & - & - & - & 4 & 10,3 & 8 & 16,0 \\
\hline $\begin{array}{l}\text { Aspiración laríngea con defensa } \\
\text { efectiva }\end{array}$ & - & - & 1 & 2,6 & - & - & - & - & - & - & - & - & 1 & 2,0 \\
\hline $\begin{array}{l}\text { Aspiración traqueal con tos y } \\
\text { aclaramiento parcial }\end{array}$ & - & - & 2 & 5,1 & - & - & - & - & - & - & - & - & 2 & 4,0 \\
\hline Aspiración traqueal silente & 2 & 18,2 & 4 & 10,3 & - & - & - & - & - & - & 7 & 17,9 & 13 & 26,0 \\
\hline Total con una o más alteraciones & 52 & 18,2 & 4 & 10,3 & - & - & 7 & 17,9 & 4 & 36,4 & 25 & 64,1 & 42 & 84,0 \\
\hline
\end{tabular}


Tabla 5. Alteraciones de la fase esofágica

\begin{tabular}{|lcccccc|}
\hline Tipo de alteración & \multicolumn{2}{c}{ GMFCS I-II } & \multicolumn{2}{c}{ GMFCS III-V } & \multicolumn{2}{c|}{ Total } \\
\hline Tránsito esofágico lento & $\mathbf{n = 1 1}$ & $\mathbf{\%}$ & $\mathbf{n = 3 9}$ & $\mathbf{\%}$ & $\mathbf{n = 5 0}$ & $\mathbf{5}$ \\
\hline Reflujo gastroesofágico & 3 & 27,3 & 12 & 30,8 & 15 & 30,0 \\
\hline Total con una o ambas & - & - & 4 & 10,3 & 4 & 8,0 \\
\hline
\end{tabular}

5. Fase esofágica (Tabla 5): Las alteraciones fueron 'tránsito enlentecido' y 'reflujo gastroesofágico'. La primera afectó al $27,3 \%$ del grupo GMFCS I-II y al 30,8\% del grupo III-V, mientras que la segunda sólo se presentó en el III-V (10,3\%).

\section{Discusión}

Esta investigación determina presencia y tipo de AV en una población infantil con PC. $\mathrm{Su}$ promedio etario ronda los 5 años, edad significativa por las habilidades esperables de alimentación y deglución, y las complicaciones asociadas a su afectación ${ }^{13}$. Hay predominio masculino, acorde a datos ya conocidos para $\mathrm{PC}^{40}$. Predominan los niveles GMFCS III a V, siendo justamente los más asociados a disfagia? ${ }^{\text {. }}$

La frecuencia general de AV es alta, ya que un $94 \%$ presenta una o más anomalías en el examen. Si bien dichas alteraciones se concentran en niños GMFCS III-V, tal como informan otros autores ${ }^{18}$, llama la atención que también se observen en pacientes del grupo GMFCS I-II. Lo anterior obliga a prestarles más atención y a buscar dirigidamente alteraciones deglutorias en ellos, vía VFC, previa evaluación clínica de TAD y cuando ésta muestre banderas rojas (infecciones respiratorias recurrentes, tos o arcadas al comer/ beber, tiempos de alimentación prolongados, etc. $)^{31}$. Es importante que el personal que atiende pacientes con PC tome conciencia del problema y no suponga que el grupo GMFCS I-II está exento de sufrir trastornos deglutorios. Recientemente, un estudio menciona que en un grupo de 49 niños con PC y TAD, sólo el $20,4 \%$ había sido derivado a evaluación especializada por el equipo tratante ${ }^{33}$.
La masticación se evalúa en el $72 \%$ de la muestra. En el 28\% restante (todos GMFCS V) no se puede evaluar debido al compromiso motor. Sabido es que a mayor compromiso motor grueso, peor función oromotora, afectándose la masticación ${ }^{13,33}$. Sin embargo, cuando sea posible, ésta debe considerarse en la VFC puesto que aporta información valiosa sobre simetría deglutoria y función de cuerdas vocales ${ }^{36}$. La masticación se evalúa con proyecciones anteriores aunque en nuestro medio no siempre se logran efectuar ya que habitualmente el examen parte con proyecciones laterales, que evidencian más eficientemente una aspiración, dejándose las anteriores para el final del procedimiento, cuando el niño puede estar ya cansado para su realización. Con proyecciones laterales la masticación se estudia sólo parcialmente ya que no permiten valorar el patrón rotatorio que muestran las anteriores.

En esta investigación, las alteraciones de la eficacia deglutoria quedan bien evidenciadas por la VFC, demostrándose más frecuentes en casos GMFCS III-V, tanto en fase oral como faríngea, especialmente la primera. Dichas alteraciones no difieren mayormente de lo informado antes ${ }^{17,18,21}$, incluyendo derrame anterior por sello labial incompleto, reflujo faringonasal (el cual conduce a patología otorrinolaringológica ${ }^{13}$ ), propulsiones múltiples por alteración del sello velolingual y, destacadamente, presencia de residuos valleculares y en senos piriformes, tanto para líquidos como sólidos, constituyendo estas dos últimas, las anomalías más frecuentes del presente estudio. Éstas son altamente significativas porque constituyen un factor de riesgo mayor para aspiración postdeglución ${ }^{17}$.

La VFC también objetiva importantes problemas sobre seguridad deglutoria, similares a 
los señalados en otras publicaciones ${ }^{17,18,21}$. El derrame posterior es particularmente frecuente, sobre todo en niños GMFCS III-V, siendo una situación que favorece la aspiración ${ }^{17,20}$. Los fenómenos de penetración también exhiben alta frecuencia, ya que casi la mitad de la muestra presenta algún grado de esta alteración, especialmente los casos GMFCS III-V. Como hallazgo, esto resulta significativo porque la penetración es una señal de futura aspiración ${ }^{17,20}$. La aspiración aparece, en cualquiera de sus grados, hasta casi en un tercio del grupo analizado, frecuencia mayor a la de algunas publicaciones ${ }^{11}$, aunque similar a lo informado por otras ${ }^{20}$. La frecuencia de aspiración silente hasta el nivel traqueal, por sí misma un gran riesgo para cuadros respiratorios graves, resultó menor a lo informado por otros autores ${ }^{12,41}$, lo que podría explicarse por diferencias de objetivo y metodología entre nuestra investigación y la de sus publicaciones. Lo llamativo en este estudio es que la aspiración traqueal silente esté presente en 2 niños GMFCS I-II, en los que habitualmente no se espera. Esto obliga a considerar la evaluación clínica e instrumental de disfagia en todos los pacientes con PC, incluidos los de menor compromiso motor.

La VFC, adicionalmente, informa sobre la fase esofágica de la deglución, evidenciando alteraciones que orientan a tratamientos específicos. En este ámbito, el trastorno más común fue el tránsito esofágico lento, lo que es relevante al considerar volumen y frecuencia de aporte del alimento. El reflujo gastroesofágico es menos frecuente respecto a otras series ${ }^{11}$, posiblemente porque su diagnóstico se concreta junto a otros exámenes instrumentales que no fueron parte de esta investigación.

\section{Conclusiones}

Las AV son frecuentes en niños con PC, afectando fundamentalmente a aquellos con mayor compromiso motor. Los de compromiso motor más leve no están exentos de éstas. La VFC identifica situaciones asociadas a mayor riesgo de complicaciones en un TAD, habitualmente presentes de manera múltiple, que la evaluación clínica no necesariamente detecta. Algunas AV son silentes y pueden pasar inadvertidas si no se objetivan instrumentalmente. La frecuencia de aspiración silente, especialmente en casos de mayor compromiso motor, es un tema de importancia que amerita mayor estudio.

\section{Referencias bibliográficas}

1. Stavsky M, Mor O, Mastrolia SA, et al. Cerebral palsyTrends in epidemiology and recent development in prenatal mechanism of disease, treatment and prevention. Front Pediatr 2017; 5:21. doi: 10.3389/fped.2017.00021.

2. Rosenbaum P, Paneth N, Leviton A, et al. The definition and classification of cerebral palsy. Dev Med Child Neurol 2007; 49: 8-14.

3. Oskoui M, Coutinho F, Dykeman J, et al. An update on the prevalence of cerebral palsy: a systematic review and meta-analysis. Dev Med Child Neurol 2013; 55: 509-19.

4. Colver A, Fairhurst C, Pharoah POD. Cerebral palsy. Lancet 2014; 383: 1240-9.

5. Otapowicz D, Sobaniec W, Okurowska-Zawada B, et al. Dysphagia in children with infantile cerebral palsy. Adv Med Sci 2010; 55: 222-7.

6. Salghetti A, Martinuzzi A. Dysphagia in cerebral palsy. East Jour Med 2012; 17: 188-93.

7. Novak I. Evidence-based diagnosis, health care and rehabilitation for children with cerebral palsy. J Child Neur 2014; 29: 1141-56.

8. Ulualp S, Brown A, Shangavi R, et al. Assessment of laryngopharyngeal sensation in children with dysphagia. Laryngoscope 2013; 123: 2291-5.

9. Benfer KA, Weir KA, Boyd RN. Clinimetrics of measures of oropharyngeal dysphagia for preschool children with cerebral palsy and neurodevelopmental disabilities: a systematic review. Dev Med Child Neurol 2012; 54: 784-95

10. Erasmus CE, van Hulst K, Rotteveel JJ, et al. Clinical practice: swallowing problems in cerebral palsy. Eur J Ped 2012; 171: 409-14.

11. Seddon PC, Khan Y. Respiratory problems in children with neurological impairment. Arch Dis Child 2003; 88: 75-8.

12. Odding E, Roebroeck ME, Stam HJ. The epidemiology of cerebral palsy: incidence, impairments and risk factors. Disabil Rehabil 2006; 28: 183-91.

13. Bacco JL, Araya F, Peña N, et al. Trastornos de la ali- 
mentación y deglución en niños con parálisis cerebral: condiciones clínicas acompañantes. Rehabil.integral. 2016; 11: 25-32.

14. Jr HL, Joshi A, Lorenz Z, et al. Pediatric cerebral palsy life expectancy: has survival improved over time? Pediat Therapeut 2013; 3: 146. doi:10.4172/21610665.1000146.

15. Novak I, Morgan C, Adde L, et al. Early, accurate diagnosis and early intervention in cerebral palsy. Advances in diagnosis and treatment. JAMA Pediatr. 2017; doi:10.1001/jamapediatrics.2017.1689.

16. Weir K, McMahon S, Barry L, et al. Clinical signs and symptoms in oropharyngeal aspiration and dysphagia in children. Eur Resp J 2009; 33: 604-11.

17. Batchelor G, McNaughten B, Bourke T, et al. Arch Dis Child Educ Pract Ed 2018; 0: 1-8. doi 10.1136/archdischild-2017-313787.

18. Silva A, Piovesana A, Barcelos I, et al. Evaluación clínica y videofluoroscópica de la deglución en pacientes con parálisis cerebral tetraparésica espástica y atetósica. Rev Neurol 2006; 42: 462-5.

19. Lefton-Greif M. Pediatric Dysphagia. Phys Med Rehab Clin N Am 2008; 19: 837-51.

20. Silva-Munhoz LF, Bühler KEB, Limongi SCO. Comparison between clinical and videofluoroscopic evaluation of swallowing in children with suspected dysphagia. CoDAS 2015; 27: 186-92.

21. Lima BC, Almeida ME, Guerra A, et al. Clinical and videofluoroscopic diagnosis of dysphagia in chronic encephalopathy of childhood. Radiol Bras 2014; 47: 84-8.

22. Smith Hammond CA. Cough and aspiration of food and liquids due to oral pharyngeal dysphagia. Lung. 2008; 186 (Suppl 1): S35-40.

23. Furkim AM, Behlau MS, Weckx LL. Avaliação clínica e videofluoroscópica da deglutição em crianças com paralisia tetraparética espástica. Arq Neuropsiquiatr. 2003; 61: 611-6.

24. Arvedson JC. Assessment of pediatric dysphagia and feeding disorders: clinical and instrumental approaches. Dev Disabil Res Rev. 2008; 14: 118-27.

25. Royal College of Speech \& Language Therapists. RCSLT clinical guidelines: 5.8 Disorders of feeding, eating, drinking \& swallowing. Bicester, Speechmark Publishing Ltd., 2005; p. 65.

26. Farneti D. Disordini della deglutizione nella pratica medica ambulatoriale. Rev SIMG. 2004; 3: 23-7.

27. Rugiu MG. Role of videofluoroscopy in evaluation of neurologic dysphagia. Acta Otorhinolaryngol Ital 2007; 27: 306-16.
28. Miller CK. Updates on pediatric feeding and swallowing problems. Curr Opin Otolaryngol Head Neck Surg 2009; 17: 194-9.

29. Correia HN, Ghizoni HA, Celli A, et al. Aspiration pneumonia in children with cerebral palsy after videofluoroscopic swallowing study. Int Arch Otorhinolaryngol 2016; 20: 132-7.

30. DeMatteo C, Matovich D, Hjartarson A. Comparison of clinical and videofluoroscopic evaluation of children with feeding and swallowing difficulties. Dev Med Child Neur 2005; 47: 149-57.

31. National Institute for Health and Care Excellence (2017). Cerebral palsy under 25s: assessment and management (NICE Guideline 62) [internet]. Disponible desde: https://www.nice.org.uk/guidance/ng62/chapter/ Recommendations\#eating-drinking-and-swallowingdifficulties. Citado: Noviembre 7, 2018.

32. Dodrill P, Gosa MM. Pediatric dysphagia: physiology, assessment and management. Ann Nutr Metab 2015; 66 (suppl 5): 24-31.

33. Bacco JL, Araya F, Peña N, et al. Trastornos de la alimentación y deglución en niños con parálisis cerebral: presencia y severidad. Rehabil.integral 2016; 11: 1524.

34. American Speech-Language-Hearing Association, ASHA. Guidelines for Speech-language pathologists performing videofluoroscopic swallowing studies. 2004; 9: suppl 24: 77-92.

35. American Speech-Language-Hearing Association, ASHA. ASHA Clinical Topics: Pediatric Dysphagia. [internet] Disponible en: http://www.asha.org/PracticePortal/Clinical-Topics/Pediatric-Dysphagia/. Citado: Noviembre 7, 2018.

36. Gramigna GD. How to perform video-fluoroscopic swallowing studies. GI Mot Online. 2006. doi:10.1038/ gimo95.

37. Cámpora H, Durand L, Ané F. Trastornos de la deglución. Arch Neurol, Neurocir Neuropsiq 2001; 11: 1-8.

38. Cámpora H, Falduti A. Evaluación y tratamiento de las alteraciones de la deglución. Rev Am Med Resp 2012; 3: 98-107.

39. Rosenbek JC, Robbins JA, Roecker EB, et al. A penetration-aspiration scale. Dysphagia 1996; 11: 93-8.

40. Shapiro BK. Cerebral palsy: a reconceptualization of the spectrum. J Pediatr 2004; 145 (2S): S3-S7.

41. Kim JS, Han ZA, Song DH, et al. Characteristics of dysphagia in children with cerebral palsy, related to gross motor function. Am J Phys Med Rehabil 2013; 92 (10): 912-9. 